\title{
Effects of alternative methyl group acceptors on the growth energetics of the O-demethylating anaerobe Holophaga foetida
}

\author{
Oliver Kappler, ${ }^{1,2}$ Peter H. Janssen, ${ }^{1} \dagger$ Jan-U. Kreft ${ }^{2}$ and Bernhard Schink ${ }^{2}$
}

Author for correspondence: Oliver Kappler. Tel: +496421 178831. Fax: +496421 178809.

e-mail: kappler@mailer.uni-marburg.de

1 Max-Planck-Institut für terrestrische

Mikrobiologie, D-35043

Marburg, Germany

2 Fakultät für Biologie, Universităt Konstanz,

D-78434 Konstanz, Germany
The anaerobic bacterium Holophaga foetida can metabolize the methyl groups of methoxylated aromatic compounds either to acetate or to dimethyl sulphide. The effects of this metabolic flexibility were investigated under conditions of excess substrate (batch culture) and substrate limitation (chemostat culture). Growth yield data suggest that transfer of the methyl groups to sulphide, in contrast to the homoacetogenic transfer to $\mathrm{CO}_{2}$, was not coupled to energy conservation. Under conditions of excess substrate, methyl groups were quantitatively transferred to sulphide. Growth yields decreased but growth rates increased upon the addition of sulphide during exponential growth in pH- and sulphide-regulated batch cultures. From the measured growth yields, the Gibbs free energy dissipation of catabolism plus anabolism $\left(-\Delta G_{D}^{\circ}\right)$ was calculated using stoichiometric equations incorporating biomass formation (macrochemical equations). The observed increase in growth rate correlated well with an increase in $-\Delta G_{D}^{0}$, suggesting a relationship between growth kinetics and growth energetics. During steady-state growth in pH- and sulphide-regulated chemostat culture, a considerable fraction of the methyl groups was converted to acetate, despite the presence of sulphide. This resulted in similar growth yields and correspondingly similar $-\Delta G_{D}^{0}$ values in the presence and absence of sulphide. Apparently, $H$. foetida uncouples catabolism and anabolism in batch culture under conditions of excess substrate to a greater extent than in the chemostat under substrate limitation, by transferring the methyl groups quantitatively to sulphide and thereby dissipating the Gibbs free energy change of the methyl transfer. The physiological significance of these findings could be that $H$. foetida adjusts the energetics of its metabolism to the growth conditions (i) to maximize the growth rate if substrate is available in excess or, (ii) to maximize the growth yield if substrate is limiting.

Keywords: Holophaga foetida, Gibbs free energy dissipation, O-demethylation, sulphide methylation, syringate

\section{INTRODUCTION}

Considerable efforts have been made to develop a theoretical understanding of the coupling between substrate consumption on the one hand and biomass formation on the other (Tempest \& Neijssel, 1984; van Dam et al., 1988). The widely used, easily determinable,

†Present address: Department of Microbiology, University of Melbourne, Parkville, Victoria 3052, Australia. stoichiometric link between the exergonic processes of catabolism and the mostly endergonic processes that lead to the formation of new cell material (anabolism) is the growth yield $\left(Y_{S}\right)$ on a given substrate $(s)$. An energetic interpretation of growth yield values using the concept of $Y_{\mathrm{ATP}}$, however, poses several problems and relies on extensive biochemical knowledge of the metabolism of the organism under investigation (Tempest $\&$ Neijssel, 1984). A method requiring only 'black box information' was developed by Heijnen $\&$ van Dijken (1991). In their approach, a so-called macrochemical 
equation of the overall metabolism, which includes biomass formation, can be formulated solely from the measured growth yield values and the stoichiometry of substrate conversion to products (including biomass). Furthermore, this equation allows the calculation of the Gibbs free energy dissipation per C-mol biomass, $-\Delta G_{\mathrm{D}}^{0 \prime}$ (or $D_{\mathrm{S}}^{01} / r_{\mathrm{Ax}}$, as abbreviated by Heijnen $\&$ van Dijken, 1991), as a direct thermodynamic measure of the overall metabolism (Heijnen \& van Dijken, 1991). $-\Delta G_{\mathrm{D}}^{0 \prime}$ is equal, but opposite in sign, to the Gibbs free energy of reaction of the macrochemical equation (Heijnen \& van Dijken, 1991), and is a description of the energetics of the overall metabolism, i.e. the degree of coupling between catabolism and anabolism. Several examples show that catabolism and anabolism are often tightly coupled if energy is limiting, resulting in a maximization of growth yield (de Vries et al., 1970; Teixeira de Mattos \& Tempest, 1983), which may be a decisive factor during starvation periods. Upon changes to conditions of excess substrate, however, maximal growth rate may be decisive in competition (Neijssel \& Teixeira de Mattos, 1994). Whereas the concept of Gibbs free energy dissipation allows a thermodynamic description of growth yields, a thermodynamic description of growth kinetics is less advanced (Heijnen, 1994). A 'black box' model of microbial growth based on phenomenological non-equilibrium thermodynamics, however, predicts a linear relationship between the rate of a reaction and its driving force (i.e. the Gibbs free energy change) near and often far from equilibrium (Westerhoff \& van Dam, 1987). If this model is applicable to growth kinetics, one would expect the rate of a particular metabolism to be dependent on its driving force, i.e. $-\Delta G_{\mathrm{D}}^{\prime \prime}$.

In this investigation, the energetic and kinetic consequences of sulphide methylation on the growth of the strictly anaerobic homoacetogenic bacterium Holophaga foetida (Bak et al., 1992; Liesack et al., 1994) were studied. $H$. foetida degrades mainly methoxylated aromatic compounds such as syringate $\left[\mathrm{C}_{6} \mathrm{H}_{2} \mathrm{OH}\left(\mathrm{OCH}_{3}\right)_{2} \mathrm{COO}^{-}\right]$. The methylphenylethers are cleaved by O-demethylation (Kreft \& Schink, 1993, 1994) as suggested by Bak et al. (1992). The methyl groups can be metabolized via two pathways; either (1) being carbonylated to acetate via the acetyl-CoA pathway involving CO dehydrogenase (Kreft \& Schink, 1993), or (2) used to methylate sulphide to form methanethiol $\left(\mathrm{CH}_{3} \mathrm{SH}\right)$ and subsequently dimethyl sulphide $\left(\mathrm{CH}_{3} \mathrm{SCH}_{3}\right)$.

$$
\begin{gathered}
\mathrm{C}_{6} \mathrm{H}_{2} \mathrm{OH}\left(\mathrm{OCH}_{3}\right)_{2} \mathrm{COO}^{-}+\underset{4}{4 \mathrm{H}_{2} \mathrm{O} \rightarrow}+\mathrm{CH}_{3} \mathrm{COO}^{-}+3 \cdot 5 \mathrm{H}^{+} \\
\mathrm{C}_{6} \mathrm{H}_{2} \mathrm{OH}\left(\mathrm{OCH}_{3}\right)_{2} \mathrm{COO}^{-}+\mathrm{HS}^{-}+\mathrm{H}_{2} \mathrm{O} \rightarrow \\
3 \mathrm{CH}_{3} \mathrm{COO}^{-}+\mathrm{CH}_{3} \mathrm{SCH}_{3}+\mathrm{HCO}_{3}^{-}+2 \mathrm{H}^{+}
\end{gathered}
$$

The aromatic ring is in both cases degraded to acetate through the phloroglucinol pathway (Brune \& Schink, 1992) with concomitant energy conservation (Kreft \& Schink, 1993). Bak et al. (1992) speculated that the transfer of methyl groups to sulphide, in contrast to a homoacetogenic transfer to $\mathrm{HCO}_{3}^{-}$, might not be coupled to energy conservation, despite a preference of $H$. foetida for sulphide as methyl acceptor. This may allow the investigator to use sulphide as an experimental switch to divert methyl groups onto sulphide and thereby increase the Gibbs energy dissipation of the overall metabolism. Since the Gibbs free energy changes of methyl transfer from methylphenylethers to bicarbonate or sulphide are similar (see Discussion) this would result in a decrease in energetic efficiency of the metabolism. The addition of sulphide is thus postulated to induce a metabolism with fewer ATP conserving steps.

We developed an experimental system where the sulphide concentration as well as $\mathrm{pH}$ were monitored and regulated to study the physiological role of sulphide methylation in the metabolism of $H$. foetida. Growth yield and rate measurements in batch and continuous culture with alternative methyl group acceptors allowed a description of the growth energetics of $H$. foetida.

\section{METHODS}

Organism and growth conditions. Holophaga foetida strain TMBS4 (DSM 6591) was maintained in sulphide-reduced bicarbonate-buffered $(\mathrm{pH} 7 \cdot 0)$ freshwater mineral medium prepared under a $\mathrm{N}_{2} / \mathrm{CO}_{2}$ atmosphere $(4: 1, \mathrm{v} / \mathrm{v})$ as previously described (Bak et al., 1992). Stock cultures, with syringate $(4 \mathrm{mM})$ as sole carbon and energy source, were transferred bimonthly, incubated at $30^{\circ} \mathrm{C}$, and were stored at $4{ }^{\circ} \mathrm{C}$ in the dark. Purity was checked microscopically at each transfer.

All experiments were performed at $30^{\circ} \mathrm{C}$ in the dark in the same medium except that L-cysteine $(1 \mathrm{mM})$ was used as reducing agent, sodium sulphate $(100 \mu \mathrm{M})$ was added as sulphur source and resazurin $(4 \mu \mathrm{M})$ as redox indicator. Experiments with or without addition of sulphide were inoculated to $5 \%(\mathrm{v} / \mathrm{v})$ with cells growing exponentially in sulphide-reduced medium or cysteine-reduced medium, respectively.

In all regulated batch culture and chemostat experiments, the culture liquid was stirred with a magnetic stirring bar at approximately 300 r.p.m. Anoxia was ensured by a constant pressure $[1060 \mathrm{mbar}(106 \mathrm{kPa})]$ of the $\mathrm{N}_{2} / \mathrm{CO}_{2}(4: 1, \mathrm{v} / \mathrm{v})$ atmosphere on the system and by only using isoversinic or glass tubing. Residual oxygen was removed by passing the $\mathrm{N}_{2} / \mathrm{CO}_{2}$ gas mixture over a copper catalyst (R3-11; BASF). During batch culture experiments, the medium and outlet pumps were switched off. Batch culture and chemostat experiments were performed in two different ways. Most experiments were performed with a culture volume of about $600 \mathrm{ml}$, leaving about $500 \mathrm{ml}$ gas phase in the culture vessel. In this case the gas phase volume was not well defined because the gas phase extended into the gas supply. Some experiments were performed with a culture volume of about $1100 \mathrm{ml}$ and a defined small gas phase of about $5 \mathrm{ml}$ below the pressure lock. In this case, the gas supply to the culture vessel was disconnected. During continuous operation pump rates were checked at regular intervals by determining the flow rate at the outlet.

Sulphide and $\mathrm{pH}$ regulation. The $\mathrm{pH}$ was measured continuously with a combined electrode (405-60-S7/165; Ingold) and regulated by a two-point $\mathrm{pH} / \mathrm{mV}$ regulator $(\mathrm{M} 8832 \mathrm{~N}$; Mostec) connected to a pump supplying anoxic cysteine- 
reduced resazurin-emended $0.5 \mathrm{M} \mathrm{Na}_{2} \mathrm{CO}_{3}$ to the culture vessel. The $\mathrm{pH}$ was regulated to a value of 7.00 $\pm 0 \cdot 01$, and was checked at regular intervals by determining the $\mathrm{pH}$ in samples using an external $\mathrm{pH}$ meter. Sulphide was continuously measured as the potential between the reference electrode of the combined $\mathrm{pH}$ electrode and a home-made sulphide electrode (Cypionka, 1986), and regulated by a two-point $\mathrm{pH} / \mathrm{mV}$ regulator that controlled a pump supplying a $0.25 \mathrm{M}$ $\mathrm{Na}_{2} \mathrm{~S}$ solution to the culture vessel. The sulphide electrode was constructed by threading a piece of silver wire through a shortened $2 \mathrm{ml}$ glass pipette sealed with a small butyl rubber stopper at the lower end. A platinum wire was then temporarily connected to the upper end of the sulphide electrode while the lower ends of the electrode and the platinum wire were immersed in a $0.5 \mathrm{M}$ sulphide solution for $1 \mathrm{~h}$. This resulted in a $\operatorname{Ag}_{2} \mathrm{~S}$ coating on the silver wire. Just before inoculation of the culture sulphide electrodes were calibrated in growth medium at $30^{\circ} \mathrm{C}$ by adding defined volumes of $0.5 \mathrm{M} \mathrm{Na}_{2} \mathrm{~S}$ solution to the vessel and determining the total sulphide concentration by the methylene blue method (Cline, 1969). The electrodes showed the expected log-linear response (Cypionka, 1986) with slopes generally between 36 and $37 \mathrm{mV}$ per $\log$ (total sulphide). After calibration, the headspace of the culture vessel was flushed with $\mathrm{N}_{2} / \mathrm{CO}_{2}$ to lower the sulphide concentration to the nominal value. During growth experiments, electrodes were checked at regular intervals by sulphide determination using the methylene blue method (Cline, 1969). The inaccuracy of the sulphide regulation was generally less than $1 \mathrm{mV}$ and therefore was below the $\mathrm{mV}$ resolution of the display of the regulator. Sulphide values are therefore listed without quantifying the error (Table 2). All $\mathrm{pH} / \mathrm{mV}$ regulators were modified with additional displays and potentiometers to allow a more precise setting of the parameters. Sulphide concentrations and $\mathrm{pH}$ were continuously monitored by a chart recorder.

Sampling and analytical procedures. Liquid samples were withdrawn through a sample port without opening the culture vessel. Samples for the determination of substrate (syringate, gallate) and product (acetate) concentrations were separated from the cells in less than $0.5 \mathrm{~s}$ by sucking the culture liquid through a $0.2 \mu \mathrm{m}$ filter into a test tube, where a small vacuum was created using a $10 \mathrm{ml}$ syringe attached to a sideport. Samples were frozen immediately and stored at $-20^{\circ} \mathrm{C}$. Substrate was analysed by HPLC using a RP-18 column $(20 \mathrm{~cm} \times 4.6 \mathrm{~mm}, 4 \mu \mathrm{m}$ particle size; Grom) with acetonitrile $/ 10 \mathrm{mM}$ phosphoric acid $(11: 89, \mathrm{v} / \mathrm{v})$ as eluent, and a photometric detector at $271 \mathrm{~nm}$. Acetate was determined by ion exclusion HPLC (Krumböck \& Conrad, 1991). Immediately after sampling optical density was determined in a spectrophotometer (U-2000, Hitachi) at $578 \mathrm{~nm}$. A few crystals of sodium dithionite were added to the cuvette before each measurement to avoid colouring due to oxidation of the resazurin.

Conversion factors $\left(\mathrm{OD}_{578}\right.$ to $\mathrm{g}$ dry wt $\left.\mathrm{I}^{-1}\right)$ were determined separately for each type of experiment. Cells were harvested during exponential growth by centrifugation $(9500 \mathrm{~g})$, washed twice in ammonium acetate buffer $(50 \mathrm{mM}, \mathrm{pH} 5.3)$ and dried at $105^{\circ} \mathrm{C}$ to constant weight. When syringate was metabolized, one $\mathrm{OD}_{578}$ unit corresponded to values of $0 \cdot 3092$, $0.3623,0.3388$ and $0.3623 \mathrm{~g}_{\text {dry wt }} \mathrm{l}^{-1}$ for cells grown in regulated batch culture in the presence and absence of sulphide and those grown in continuous culture in the presence and absence of sulphide, respectively. For gallate-grown cells in regulated batch cultures, values of 0.3522 and $0.3133 \mathrm{~g}$ dry wt $\mathrm{I}^{-1}$ per $\mathrm{OD}_{578}$ unit were determined in the presence and absence of sulphide, respectively.
Samples for sulphide determination were added to an aliquot of $2 \%(\mathrm{w} / \mathrm{v})$ zinc acetate directly after removal from the culture vessel. Due to interference of the methylene blue method with components of the medium, the resulting zinc sulphide suspensions were washed by centrifugation and resuspension in fresh zinc acetate solution. Contrary to the results of Finster et al. (1992), methanethiol and dimethyl sulphide did not interfere with colour formation in the test. Methanethiol, however, was precipitated by zinc and, therefore, probably lowered the available zinc ion concentration.

Gas samples $(0 \cdot 2 \mathrm{ml})$ for determination of volatile organosulphur compounds (dimethyl sulphide, methanethiol) were withdrawn from the headspace of the culture vessel through a pressure lock Teflon stopper (Dynatech Mininert) using a pressure lock syringe, and were analysed by gas chromatography with a Chromosil 330 column (Supelco) and a flame ionization detector. The temperatures were $70^{\circ} \mathrm{C}$ for the column, and $130^{\circ} \mathrm{C}$ for injector and detector. Concentrations of methanethiol and dimethyl sulphide in the liquid phase were calculated from the concentrations measured in the gas phase of the culture system (Bak et al., 1992).

\section{Calculations}

Batch culture experiments. The sulphide- and $\mathrm{pH}$-regulated batch culture system was developed to study growth under conditions of excess substrate. Therefore, only data points (generally at least five) during the exponential growth phase of the culture were used (Fig. 1). This phase was identified by loglinear regression of biomass concentration versus time, yielding the growth rate $(\mu)$ as the regression coefficient. The molar growth yield $\left(Y_{\mathrm{s}}\right)$ with substrate $s$ was determined as the regression coefficient of a linear regression of biomass concentration versus substrate concentration during exponential growth (Fig. 1b). The specific substrate consumption rate $\left(q_{\mathrm{S}}\right)$, defined as the differential change in substrate $s$ with time $t$ normalized to the biomass $(x)$ concentration $\left(q_{\mathrm{s}}=1 / x \mathrm{~d} s / \mathrm{d} t\right)$, was calculated using the relationship $\mu=Y_{\mathrm{S}} \cdot q_{\mathrm{S}}$. This is, however, only possible if $\mu$ and $Y_{s}$ are constant, which was shown to be the case in exponentially growing batch cultures (Fig. 1) or during steadystate growth in continuous culture.

Continuous culture experiments. The sulphide- and $\mathrm{pH}$-regulated continuous culture system was developed to study growth under conditions of substrate limitation. Therefore, only data points during steady state were used for calculations. Establishment of a steady state was assumed only after at least five volume changes of the culture vessel and if only minor variations occurred in the measured variables $(\mathrm{pH}$, sulphide concentration, biomass concentration, substrate concentration, acetate concentration). The same dilution rate $\left(0.03 \mathrm{~h}^{-1}\right)$ was chosen for all experiments. Samples were routinely collected at daily intervals. $Y_{s}$ was calculated as the mean of the growth yield values determined for separate samples collected during steady state. The number of data points was five or greater in all experiments. The specific substrate consumption rate $\left(q_{\mathrm{s}}\right)$ was calculated as described above for exponential growth.

Determination of conversion stoichiometries. The stoichiometry of the metabolism allows an estimation of the relative importance of each of the two possible pathways of methyl group metabolism, i.e. sulphide methylation versus acetate formation. The ratio of acetate produced per syringate consumed should be 4.5 if methyl groups are metabolized only via the acetyl-CoA pathway, and 3 if the methyl groups are quantitatively transferred to sulphide [see Introduction, 

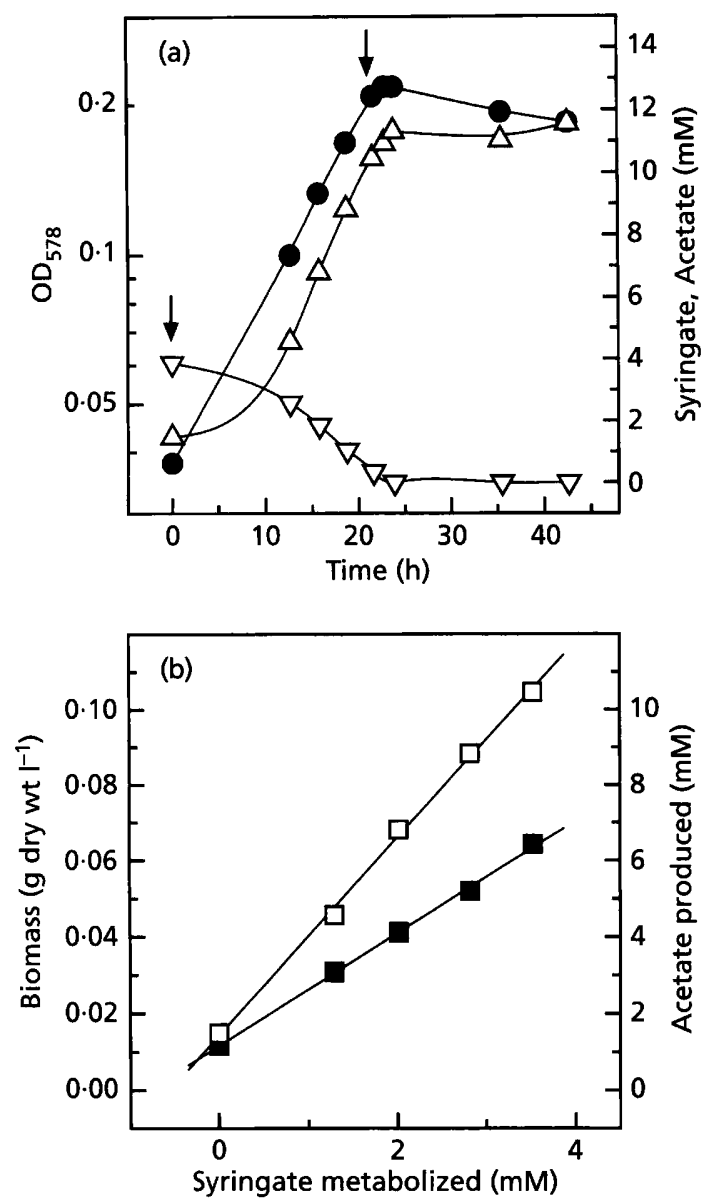

Fig. 1. (a) Growth curve of $H$. foetida, showing the increase in biomass $\left(O D_{578}, 0\right)$ coupled to the consumption of syringate $(\nabla)$ and the production of acetate $(\Delta)$. The first and the last points of the exponential growth phase are marked with arrows. (b) Determination of growth yield and acetate/syringate ratios during the exponential growth phase by linear regression of biomass produced versus syringate metabolized ( $\boldsymbol{D}$ ) and acetate produced versus syringate metabolized $(\square)$, respectively.

equations (1) and (2)]. The stoichiometric relationship between catabolic end-products produced (acetate, dimethyl sulphide, methanethiol) and substrate consumed during exponential growth in regulated batch culture was determined as the regression coefficient of a linear regression of product concentration versus substrate concentration [see for example the acetate/syringate ratio in Fig. 1(b)]. In continuous culture experiments, this stoichiometry was calculated from the ratio of the acetate concentration and the difference between the substrate concentration in the reservoir and in the culture vessel during steady state. Acetate was assumed to be the anabolic substrate and acetate assimilation into cell carbon was calculated from the growth yield with biomass expressed in $\mathrm{C}$-mol $\left(\left\langle\mathrm{C}_{1} \mathrm{H}_{1 \cdot 75} \mathrm{O}_{0.75}\right\rangle\right)$ using the anabolic equation proposed by Schink \& Pfennig (1982):

$$
\begin{aligned}
& 17 \mathrm{CH}_{3} \mathrm{COO}^{-}+17 \mathrm{H}^{+} \rightarrow \\
& 32\left\langle\mathrm{C}_{1} \mathrm{H}_{1 \cdot 75} \mathrm{O}_{0 \cdot 75}\right\rangle+2 \mathrm{HCO}_{3}^{-}+2 \mathrm{H}^{+}+4 \mathrm{H}_{2} \mathrm{O}
\end{aligned}
$$

The total amount of acetate formed is the sum of the net acetate produced and the acetate assimilated in anabolism.
Calculation of macrochemical equations and thermodynamic parameters. The macrochemical equation is a description of the stoichiometry of the metabolism that takes into account both catabolic and anabolic reactions. In this communication, the macrochemical equations (Table 3 ) were formulated solely from the measured growth yields and the stoichiometry of substrate conversion to products (Heijnen \& van Dijken, 1991). From the growth yield, a stoichiometric relationship between substrate and biomass could be formulated using the formula for biomass $\left\langle\left\langle\mathrm{C}_{1} \mathrm{H}_{1 \cdot 75} \mathrm{O}_{0.75}\right\rangle\right.$; see above $)$. All other stoichiometric coefficients were calculated using conservation equations for $\mathrm{C}, \mathrm{H}, \mathrm{O}$ and electric charge. The resulting macrochemical equation was used to calculate the Gibbs free energy dissipation of the process $\left(-\Delta G_{\mathrm{D}}^{0 \prime}\right.$ or $D_{\mathrm{s}}^{01} / r_{\mathrm{Ax}}$, Heijnen \& Dijken, 1991; Table 3) which is equal, but opposite in sign, to the Gibbs free energy change of this reaction $\left(-\Delta G^{0 \prime}\right)$. The $-\Delta G_{\mathrm{f}}^{0^{\prime}}$ of syringate $\left(-452 \mathrm{~kJ} \mathrm{~mol}^{-1}\right)$ was calculated from a tabulated value for $p$-hydroxybenzoate (Thauer et al., 1977) using a group contribution method (Mavrovouniotis, 1991), which was also used to calculate the $-\Delta G^{0 \prime}$ of the methyl transfer reactions (see Discussion). All other $-\Delta G_{\mathrm{p}}^{0^{\prime}}$ values were from published tables (Thauer et al., 1977; Heijnen \& van Dijken, 1991).

Statistics. Regressions were done as least squares analyses. The statistical significance of differences between mean values was tested with the Student's $t$-test $(\alpha=0 \cdot 01)$.

\section{RESULTS}

\section{Optimization of growth conditions}

In preliminary experiments, we studied the dependence of $\mu$ and growth yields ( $\triangle \mathrm{OD}$ ) on the syringate concentration and found maximal $\mu$ and $\Delta O D$ proportional to the amount of substrate utilized at initial syringate concentrations of up to $4 \mathrm{mM}$. Syringate at a concentration of $8 \mathrm{mM}$ resulted in a lower $\mu$ and an increase in $\triangle \mathrm{OD}$ that was no longer proportional to the initial substrate concentration. At an initial syringate concentration of $12 \mathrm{mM}$ only linear growth and a decrease in $\triangle \mathrm{OD}$ were observed. Therefore, $4 \mathrm{mM}$ syringate was routinely added to all regulated batch and chemostat experiments as a concentration that supported the maximal $\mu$ and resulted in high $\Delta \mathrm{OD}$, but did not cause any detectable inhibition.

Growth with syringate in cysteine-reduced medium supported only linear growth after two transfers when no extra sulphur source was added. However, growth did not cease, even after seven subsequent transfers. The reason for this might be that sulphide becomes available to the organism at a low rate by chemical degradation of cysteine. The addition of sulphide $(100 \mu \mathrm{M})$, thiosulphate $(50 \mu \mathrm{M})$, or sulphate $(100 \mu \mathrm{M})$ as additional sulphur sources supported exponential growth at equally high rates.

\section{Stoichiometry of metabolism}

From a $\mathrm{pH}$ - and sulphide-regulated batch culture experiment with a culture volume of approximately $1100 \mathrm{ml}$ and a small gas phase of approximately $5 \mathrm{ml}$ that was not connected to the gas supply, we determined 
Table 1. Product formation and carbon balance of $H$. foetida grown in the presence of sulphide with excess syringate as substrate

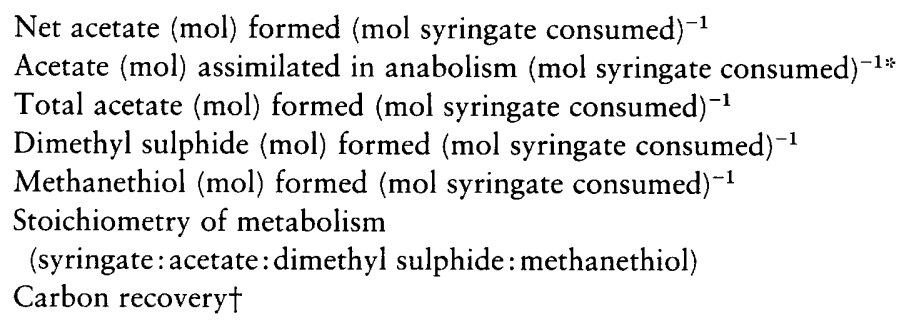

$2 \cdot 68 \pm 0.061$

$0 \cdot 33 \pm 0 \cdot 007$

$3 \cdot 01$

$1 \cdot 09 \pm 0 \cdot 040$

$0 \cdot 099 \pm 0.0029$

$1 \cdot 0: 3 \cdot 01: 1 \cdot 09: 0 \cdot 10$

$103 \%$

*Calculated from the growth yield of $15.88 \mathrm{~g}$ dry wt (mol substrate) ${ }^{-1}$ using equation (3) (see Methods).

†Ratio of C-mol products and C-mol substrate. Bicarbonate was assumed to be formed stoichiometrically from syringate [see Introduction, equation (2)].

the stoichiometric relationship of the substrate and products and calculated a carbon balance (Table 1). This stoichiometry of metabolism as well as the carbon balance revealed that the methyl groups were quantitatively transferred to sulphide and that the predominant methylated sulphur compound produced was dimethyl sulphide (Table 1). In a subsequent chemostat experiment, for as yet unknown reasons, no steady state was reached even after 13 volume changes. When the system was operated with only $600 \mathrm{ml}$ culture liquid and a larger gas phase of $500 \mathrm{ml}$ in the culture vessel, a steady state was reached after about five volume changes. Therefore, all further regulated batch and chemostat experiments were done with the smaller culture volume and consequently with a gas phase volume which was insufficiently defined for the quantification of volatile sulphur compounds because it was connected to the gas supply. Therefore, the acetate/syringate ratio was used as a measure of the relative importance of the two possible pathways of methyl group metabolism. Biomass, acetate, dimethyl sulphide and methanethiol were the only products detected. A transient accumulation of intermediates such as 5-hydroxyvanillate $(4,5$ dihydroxy-3-methoxybenzoate) or gallate $(3,4,5-$ trihydroxybenzoate) was not observed.

\section{Sulphide and $\mathrm{pH}$-regulated batch culture}

Sulphide addition has a major effect on growth rate, growth yield and substrate consumption if substrate is in excess. We found that the growth rate was significantly higher if sulphide was added to the medium. A mean increase in $\mu$ by a factor of $2 \cdot 2$ (Table 2 , column 2) was calculated from six independent experiments. The growth yield in the presence of sulphide decreased significantly, to about half that obtained in the absence of sulphide (Table 2, column 3). Specific substrate consumption rates $\left(q_{\mathrm{S}}\right)$ during exponential growth were calculated according to the equation $q_{\mathrm{S}}=\mu / Y_{\mathrm{S}}$ because $\mu$ and $Y_{\mathrm{S}}$ were constant during this growth phase (Fig. 1). On average, a fourfold increase in $q_{\mathrm{S}}$ in the presence of sulphide was calculated (Table 2, column 4). Sulphide apparently has a significant influence on the growth of $H$. foetida if the methoxylated substrate syringate is used, by increasing the specific substrate consumption rate and the specific growth rate, but decreasing the growth yield.

In control experiments we tested whether sulphide influenced growth with the non-methylated substrate gallate. Gallate supported growth at very high rates but with unpredictable lag phases (hours up to months). The sulphide concentration in these experiments was monitored but not regulated, to test for sulphideutilizing processes unrelated to methyl group metabolism. In the experiment shown (Table 2), the gallate concentration decreased by $3.14 \mathrm{mM}$, whereas the sulphide concentration was only $0.3 \mathrm{mM}$ lower at the end of the experiment. In contrast to the results with the methoxylated compound syringate, sulphide had little effect on the $Y_{S}$ values (Table 2, column 3). The specific growth rate $(\mu)$ and the specific substrate consumption rate $\left(q_{\mathrm{s}}\right)$, however, were $1 \cdot 3$-fold and $1 \cdot 2$-fold higher, respectively, when sulphide was added (Table 2, columns 2 and 4 ). The significance of the latter finding is not clear. Due to the major experimental obstacles with gallate, no further experiments were carried out with this substrate.

Sulphide methylation predominates in methyl group metabolism. The acetate/syringate ratios obtained in experiments where sulphide was added (Table 2, column 7) were close to the value of 3 that was to be expected if all methyl groups were transferred to sulphide. When no sulphide was added, the acetate/syringate ratios were close to the value of 4.5 that would be expected if all the methyl groups were transferred to $\mathrm{HCO}_{3}^{-}$(Table 2, column 7). Sulphide addition apparently led to diversion of methyl groups to sulphide under conditions of excess substrate, to form predominantly dimethyl sulphide.

\section{Sulphide and pH-regulated continuous culture}

Generally, continuous culture experiments were inoculated as a regulated batch culture and continuous operation was started during exponential growth. At the 
Table 2. Influence of sulphide on growth parameters of $H$. foetida grown with excess substrate (batch culture) and under substrate limitation (continuous culture)

Values with SD (columns 2, 3, 5, and 6) were determined as (i) slopes of linear regressions (batch cultures) or (ii) means of data points under steady-state conditions (continuous culture). Generally the number of data points was five or greater. The initial substrate concentration in regulated batch culture experiments as well as the substrate concentration in the medium reservoir of the continuous culture was approximately $4 \mathrm{mM}$.

\begin{tabular}{|c|c|c|c|c|c|c|}
\hline \multirow[b]{2}{*}{$\begin{array}{c}1 \\
\text { Sulphide* } \\
(\mathbf{m M})\end{array}$} & \multirow[b]{2}{*}{$\begin{array}{c}2 \\
\boldsymbol{\mu}_{\dagger}^{\dagger} \\
\left(\mathbf{h}^{-1}\right)\end{array}$} & \multirow[b]{2}{*}{$\begin{array}{c}3 \\
Y_{\mathrm{s}} \ddagger \\
{\left[\begin{array}{c}\text { [g dry wt }(\mathbf{m o l} \\
\left.\text { substrate })^{-1}\right]\end{array}\right.}\end{array}$} & \multirow[b]{2}{*}{$\begin{array}{c}4 \\
q_{s} S \\
{\left[\mathrm{mmol} \mathrm{h}^{-1}\right.} \\
\left.(\mathrm{g} \text { dry wt })^{-1}\right]\end{array}$} & \multicolumn{3}{|c|}{ Stoichiometry of acetate production } \\
\hline & & & & $\begin{array}{c}5 \\
\text { Net acetate } \\
\text { production } \\
\text { [mol acetate } \\
\text { produced } \\
\langle\text { (mol substrate } \\
{\left.\text { consumed })^{-1}\right]}^{\text {cons }}\end{array}$ & $\begin{array}{c}\mathbf{6} \\
\text { Anabolism } \| \\
{[\text { mol acetate }} \\
\text { assimilated } \\
\text { (mol substrate } \\
\left.\text { consumed })^{-1}\right]\end{array}$ & $\begin{array}{c}7 \\
\text { Total acetate } \\
\text { production } \\
\text { (columns } 5+6 \text { ) } \\
\text { [mol acetate } \\
\text { produced } \\
\text { (mol substrate }^{\left.\text {consumed })^{-1}\right]}\end{array}$ \\
\hline \multicolumn{7}{|c|}{ Regulated batch culture with syringate } \\
\hline 0.06 & $0.085 \pm 0.0039$ & $14 \cdot 7 \pm 0 \cdot 32$ & $5 \cdot 78$ & $2.57 \pm 0.05$ & $0 \cdot 303 \pm 0 \cdot 0066$ & $2 \cdot 9 \pm 0 \cdot 05$ \\
\hline 0.08 & $0.085 \pm 0.0016$ & $14 \cdot 0 \pm 0 \cdot 45$ & 6.07 & $2 \cdot 94 \pm 0 \cdot 10$ & $0 \cdot 288 \pm 0.0093$ & $3 \cdot 2 \pm 0 \cdot 10$ \\
\hline $0 \cdot 30$ & $0 \cdot 104 \pm 0 \cdot 0005$ & $14.3 \pm 0.55$ & $7 \cdot 27$ & $2 \cdot 74 \pm 0.09$ & $0 \cdot 296 \pm 0 \cdot 0114$ & $3 \cdot 0 \pm 0.09$ \\
\hline- & $0.034 \pm 0.0003$ & $25 \cdot 0 \pm 0 \cdot 48$ & $1 \cdot 36$ & $3 \cdot 80 \pm 0 \cdot 10$ & $0.525 \pm 0.0098$ & $4 \cdot 3 \pm 0 \cdot 10$ \\
\hline- & $0.038 \pm 0.0009$ & $26 \cdot 5 \pm 0 \cdot 38$ & $1 \cdot 43$ & $3 \cdot 86 \pm 0 \cdot 05$ & $0.547 \pm 0.0078$ & $4 \cdot 4 \pm 0.05$ \\
\hline- & $0.054 \pm 0.0003$ & $29 \cdot 1 \pm 1 \cdot 00$ & $1 \cdot 86$ & $3 \cdot 94 \pm 0 \cdot 26$ & $0 \cdot 600 \pm 0.0206$ & $4 \cdot 5 \pm 0 \cdot 26$ \\
\hline \multicolumn{7}{|c|}{ Regulated batch culture with gallate } \\
\hline $0 \cdot 41-0 \cdot 14$ & $0 \cdot 132 \pm 0 \cdot 0029$ & $11 \cdot 7 \pm 0 \cdot 48$ & $11 \cdot 28$ & $2.45 \pm 0.08$ & $0 \cdot 241 \pm 0.0098$ & $2 \cdot 7 \pm 0.08$ \\
\hline- & $0.098 \pm 0 \cdot 0016$ & $10 \cdot 7 \pm 0 \cdot 27$ & $9 \cdot 16$ & $2 \cdot 56 \pm 0.07$ & $0 \cdot 221 \pm 0 \cdot 0055$ & $2 \cdot 8 \pm 0.07$ \\
\hline \multicolumn{7}{|c|}{ Continuous culture with syringate } \\
\hline $0 \cdot 08$ & 0.03 & $21 \cdot 7 \pm 0 \cdot 11$ & $1 \cdot 38$ & $3 \cdot 18 \pm 0 \cdot 069$ & $0.448 \pm 0.0022$ & $3.6 \pm 0.07$ \\
\hline $1 \cdot 08-2 \cdot 15$ & 0.03 & $21 \cdot 3 \pm 0 \cdot 42$ & $1 \cdot 41$ & $3 \cdot 18 \pm 0 \cdot 058$ & $0.440 \pm 0.0087$ & $3.6 \pm 0.06$ \\
\hline- & $0 \cdot 03$ & $24 \cdot 5 \pm 0 \cdot 21$ & $1 \cdot 22$ & $3 \cdot 86 \pm 0 \cdot 154$ & $0.505 \pm 0.0042$ & $4 \cdot 4 \pm 0 \cdot 15$ \\
\hline
\end{tabular}

*Sulphide concentration determined as total sulphide $\left(\mathrm{H}_{2} \mathrm{~S}+\mathrm{HS}^{-}+\mathrm{S}^{2-}\right)$.

† Specific growth rate; for continuous culture experiments, the value of the dilution rate $(D)$ is given.

$\ddagger$ Molar growth yield on substrate $s$.

SSpecific consumption rate of substrate $s$, calculated from columns 2 and 3 according to $\mu=Y_{\mathrm{S}} \cdot q_{\mathrm{S}}$; sD values were $0 \cdot 005 \%$ or less of the tabulated mean in all cases.

|| Calculated from column 3, using equation (3) (see Methods).

end of one continuous culture experiment in the presence of sulphide, about $90 \%$ of the volume in the culture vessel was replaced with fresh medium to start another regulated batch culture experiment, also with sulphide addition. The growth kinetics, growth yield and stoichiometries did not differ from those obtained before continuous operation.

Sulphide addition has little effect on growth yield and substrate consumption under substrate limitation. Sulphide addition, even to millimolar concentrations, had little effect on $Y_{S}$ (Table 2, column 3) in continuous culture. Consequently, the calculated $q_{\mathrm{S}}$ values increased slightly but not statistically significantly in the presence of sulphide. This is in stark contrast to batch cultures, where sulphide had a marked effect on $Y_{S}$. In the absence of sulphide, $Y_{\mathrm{S}}$ values were similar in batch and continuous culture (Table 2, column 3 ).

A considerable portion of the methyl groups is metabolized via the acetyl-CoA pathway. The acetate/syringate ratio in continuous culture without addition of sulphide was close to the value of 4.5 that would be expected if all methyl groups were channelled into the acetyl-CoA pathway. Upon the addition of 0.08 and $1.08-2 \cdot 15 \mathrm{mM}$ sulphide, a stoichiometry of 3.6 was found (Table 2 , column 7). This ratio is significantly higher than the mean value upon addition of sulphide in batch culture experiments (3.03), and lower than the values for cultures without addition of sulphide in regulated batch and continuous culture experiments $(4 \cdot 40)$. Under conditions of substrate limitation, a considerable part of 
the methyl groups is apparently channelled into the acetyl-CoA pathway, even in the presence of millimolar concentrations of sulphide.

\section{DISCUSSION}

From the carbon balances and acetate/syringate ratios in regulated batch culture (Tables 1 and 2), we conclude that, upon addition of sulphide under conditions of excess substrate, the methyl groups of the methoxylated aromatic substrate syringate were quantitatively transferred to sulphide to form predominantly dimethyl sulphide. In the absence of sulphide, the methyl groups were metabolized to acetate (Table 2). We did not detect any aromatic intermediates, in contrast to some homoacetogenic bacteria (Frazer, 1994) or end-products other than biomass, acetate, methanethiol and dimethyl sulphide. Therefore, sulphide addition resulted in a diversion of the methyl groups from the acetyl-CoA pathway to the sulphide methylation pathway. A concomitant mean decrease in growth yield of $6.27 \mathrm{~g}$ dry wt per mol methyl group metabolized was observed (Table 2 column 3). In contrast, cultures growing with the non-methoxylated substrate, gallate, showed no significant decrease in growth yield upon sulphide addition, indicating that sulphide addition has a specific effect on energy conservation in methyl group metabolism. The growth yield differences with and without sulphide correspond well with growth yields on syringate and other methoxylated aromatic compounds determined for the homoacetogenic bacterium Acetobacterium woodii $(5 \cdot 3-6 \cdot 1 \mathrm{~g}$ dry wt per mol methyl group metabolized in the absence of yeast extract; Bache \& Pfennig, 1981; Kreikenbohm, 1984; Tschech \& Pfennig, 1984). A. woodii, in contrast to $H$. foetida, does not ferment the aromatic ring and is, therefore, restricted to energy conservation by metabolism of the methyl groups via the acetyl-CoA pathway. These considerations suggest that sulphide methylation in $H$. foetida may not be coupled to energy conservation. Thermodynamically, however, energy conservation is possible, since the Gibbs free energy changes of sulphide methylation and acetate formation are similar, with $\Delta G^{\prime \prime}$ values per mol methyl group of $-54 \mathrm{~kJ}$ and $-66 \mathrm{~kJ}$, respectively, and sufficiently negative to allow biological energy conservation. In methanogens, the transfer of a methyl group from methyl-tetrahydromethanopterin to a thiol (coenzyme $M$ ) can be used to generate a transmembrane sodium gradient (Becher et al., 1992) which shows that energy conservation is mechanistically possible. Apparently, $H$. foetida lacks a metabolic mechanism to conserve energy in this exergonic reaction and wastes the energy of the methylether bond if grown under conditions of excess substrate.

The growth rates in regulated batch cultures varied somewhat under apparently identical experimental conditions. The reason for this is at present not clear, but several possibilities could be excluded: (i) addition of dithionite to quickly remove traces of oxygen had no effect, (ii) different stirring rates did not influence growth, nor (iii) did the addition of a reductant to the sodium carbonate solution used in the $\mathrm{pH}$-regulating circuit. A $q_{\mathrm{S}}$ versus $\mu$ plot revealed, however, a linear relationship between the two parameters. This indicates that $Y_{\mathrm{S}}$ and $q_{\mathrm{S}}$ vary systematically with $\mu$, as predicted by Pirt's model (Pirt, 1965). To investigate this point further, chemostat experiments at different dilution rates are planned.

What could be the advantage of sulphide methylation in $H$. foetida? Since sulphide is the preferred methyl acceptor in batch culture (this study) and is metabolized to depletion (Bak et al., 1992), it is unlikely that sulphide methylation is an unspecific side reaction of the demethylating system. Furthermore, the reaction is probably not a detoxification mechanism like that proposed for the $S$-adenosylmethionine-dependent sulphide methylation by the ciliate Tetrahymena thermophila (Drotar et al., 1987), since (i) H. foetida grows without being adversely affected in sulphidereduced medium with non-methylated compounds (Bak et al., 1992; this study), (ii) growth in continuous culture was apparently not negatively influenced by sulphide concentrations up to $2 \mathrm{mM}$, and (iii) the specific substrate consumption rate in cell suspension experiments was not adversely affected up to $4 \mathrm{mM}$ sulphide (unpublished results).

The presence of sulphide results not only in a decrease in growth yield to about half of that in its absence, but also in a fourfold increase in the calculated specific substrate consumption rate and a $2 \cdot 2$-fold increase in the specific growth rate. The decrease in growth yield upon transfer of the methyl groups to sulphide suggests that less ATP is synthesized per mol substrate consumed. At the same time, however, the specific substrate consumption rate is increased to such an extent that more cell material is formed per unit time. Thus, the specific rate of ATP synthesis may be increased by sulphide methylation under conditions of excess substrate.

The diversion of methyl groups from the acetyl-CoA pathway to the sulphide methylation pathway under conditions of excess substrate might be interpreted in kinetic terms. From the fact that the specific substrate consumption rate in the presence of sulphide is about fourfold higher, it might be concluded that the reactions of the acetyl-CoA pathway allow a flux lower than those of the sulphide methylation pathway. In this case the overall flux would be limited mainly by the flux through the acetyl-CoA pathway. An increase in flux through the phloroglucinol (ring-degrading) pathway with a concomitant increase in the rate of ATP formation would therefore be possible if methyl groups were transferred to sulphide to increase the rate of gallate production, the putative substrate (Brune \& Schink, 1992; Kreft \& Schink, 1993) of the phloroglucinol pathway. In this case one would still expect methyl groups to be metabolized through the acetyl-CoA pathway at a rate similar to that of cultures growing in the absence of sulphide. The acetate/syringate ratios (Table 2 ) during exponential 
Table 3. Stoichiometric coefficients and Gibbs free energy dissipation values $\left(-\Delta G_{D}^{0}\right)$ of macrochemical equations calculated from experimentally determined yield values

Equations of the form

$-\mathrm{aC}_{6} \mathrm{H}_{2} \mathrm{OH}\left(\mathrm{OCH}_{3}\right)_{2} \mathrm{COO}^{-}-\mathrm{bHS}^{-}-\mathrm{cH}_{2} \mathrm{O}+1\left\langle\mathrm{C}_{1} \mathrm{H}_{1 \cdot 75} \mathrm{O}_{0 \cdot 75}\right\rangle+\mathrm{bCH}_{3} \mathrm{SCH}_{3}+\mathrm{dCH}_{3} \mathrm{COO}^{-}+\mathrm{eHCO}_{3}^{-}+\mathrm{fH}^{+}=0$ and

$-\mathrm{aC}_{6} \mathrm{H}_{2} \mathrm{OH}\left(\mathrm{OCH}_{3}\right)_{2} \mathrm{COO}^{-}-\mathrm{cH}_{2} \mathrm{O}+1\left\langle\mathrm{C}_{1} \mathrm{H}_{1 \cdot 75} \mathrm{O}_{0 \cdot 75}\right\rangle+\mathrm{dCH}_{3} \mathrm{COO}^{-}+\mathrm{eHCO}_{3}^{-}+\mathrm{fH}^{+}=0$

were used for metabolism in the presence and absence of sulphide, respectively.

\begin{tabular}{|c|c|c|c|c|c|c|c|c|c|}
\hline \multirow{2}{*}{$\begin{array}{c}\text { Total } \\
\text { sulphide } \\
(\mathrm{mM})\end{array}$} & \multicolumn{2}{|c|}{$\mathbf{Y}_{\mathrm{S}}$} & \multicolumn{6}{|c|}{ Stoichiometric coefficients } & \multirow{2}{*}{$\begin{array}{c}-\Delta G_{\mathrm{D}}^{0 \prime} \\
{[\mathrm{kJ}} \\
(\mathrm{C}-\mathrm{mol} \\
\left.\text { biomass })^{-1}\right]\end{array}$} \\
\hline & $\begin{array}{l}\text { [g dry wt (mol } \\
\text { syringate })^{-1} \text { ] }\end{array}$ & $\begin{array}{c}{[\mathrm{C} \text {-mol }} \\
\text { biomass } \\
(\mathrm{C}-\mathrm{mol} \\
\left.\text { substrate })^{-1}\right]\end{array}$ & $\begin{array}{c}\text { a } \\
\text { syringate }\end{array}$ & $\begin{array}{c}\text { b } \\
\text { sulphide }\end{array}$ & $\begin{array}{c}c \\
\mathrm{H}_{2} \mathrm{O}\end{array}$ & $\begin{array}{c}\mathrm{d} \\
\text { acetate }\end{array}$ & $\stackrel{\mathrm{e}}{\mathrm{HCO}_{3}^{-}}$ & $\begin{array}{c}\mathbf{f} \\
\mathrm{H}^{+}\end{array}$ & \\
\hline \multicolumn{10}{|c|}{ Regulated batch culture with syringate } \\
\hline 0.06 & $14 \cdot 7$ & 0.063 & 1.752 & 1.752 & $6 \cdot 882$ & $4 \cdot 724$ & $1 \cdot 814$ & $3 \cdot 035$ & 581 \\
\hline 0.08 & $14 \cdot 0$ & $0 \cdot 060$ & $1 \cdot 839$ & $1 \cdot 839$ & $7 \cdot 232$ & $4 \cdot 987$ & 1.902 & $3 \cdot 210$ & 614 \\
\hline $0 \cdot 3$ & $14 \cdot 3$ & $0 \cdot 062$ & $1 \cdot 801$ & $1 \cdot 801$ & $7 \cdot 078$ & $4 \cdot 871$ & $1 \cdot 863$ & $3 \cdot 133$ & 599 \\
\hline- & $25 \cdot 0$ & $0 \cdot 108$ & $1 \cdot 030$ & - & 3.995 & $4 \cdot 104$ & $0 \cdot 063$ & $3 \cdot 136$ & 332 \\
\hline - & $26 \cdot 5$ & $0 \cdot 114$ & $0 \cdot 972$ & - & 3.762 & $3 \cdot 841$ & 0.063 & $2 \cdot 932$ & 308 \\
\hline- & $29 \cdot 1$ & $0 \cdot 126$ & $0 \cdot 885$ & - & $3 \cdot 415$ & $3 \cdot 451$ & 0.063 & $2 \cdot 628$ & 273 \\
\hline \multicolumn{10}{|c|}{ Continuous culture with syringate } \\
\hline $0.08 \%$ & 21.7 & 0.094 & $1 \cdot 187$ & 0.690 & $4 \cdot 622$ & $3 \cdot 773$ & 0.753 & $2 \cdot 649$ & 379 \\
\hline $1 \cdot 08-2 \cdot 15^{*}$ & $21 \cdot 3$ & 0.092 & $1 \cdot 209$ & $0 \cdot 710$ & $4 \cdot 711$ & $3 \cdot 844$ & 0.772 & $2 \cdot 698$ & 387 \\
\hline- & $24 \cdot 5$ & $0 \cdot 106$ & $1 \cdot 051$ & - & $4 \cdot 079$ & $4 \cdot 198$ & 0.063 & $3 \cdot 210$ & 340 \\
\hline
\end{tabular}

* The stoichiometric coefficients for acetate were calculated using the experimentally determined values (Table 2, column 5).

growth in batch culture, however, are close to the value of 3 that is expected if acetate is solely produced via the phloroglucinol pathway and the contribution of the acetyl-CoA pathway to acetate production is insignificant. A kinetic explanation, that a saturation of the acetyl-CoA pathway under conditions of excess substrate leads to the diversion of methyl groups to sulphide, therefore seems unlikely.

To compare the metabolism of $H$. foetida in the presence and absence of sulphide in thermodynamic terms, a macrochemical equation was formulated (Heijnen \& van Dijken, 1991) to calculate the corresponding Gibbs free energy dissipation $\left(-\Delta G_{\mathrm{D}}^{0 \prime} ;\right.$ Table 3$)$. The measured net acetate formation in the experiments carried out (Table 2, column 5) differed from the values predicted by the macrochemical equation (Table 3) only by $-7 \cdot 8 \%$ to $4.9 \%$ suggesting that $-\Delta G_{\mathrm{D}}^{0 \prime}$ is a suitable measure of the energetics of the metabolism of $H$. foetida. Interestingly, the doubling in $-\Delta G_{\mathrm{D}}^{0^{\prime}}$ upon addition of sulphide correlated well with a $2 \cdot 2$-fold increase in $\mu$, making it tempting to speculate about a proportional relationship between energy dissipation and growth rate.

The relationships between thermodynamics and growth kinetics are at present not very well understood (Heijnen, 1994). A possible description of the apparent proportionality between dissipated energy and growth rate in $H$. foetida may, however, be deduced from phenomenological non-equilibrium thermodynamics applied to biological systems (Stucki, 1980; van Dam et al., 1988). In this 'black box' description, the cell is viewed, much like a steam engine, as a linear energy converter where catabolic (energy-releasing) processes are coupled to a certain degree to anabolic (energyconsuming) processes. From the second law of thermodynamics, it follows that coupling has to be incomplete to create a positive flux which allows the system to perform work. This means that a positive substrate consumption rate is possible only if less than $100 \%$ of the Gibbs free energy change of catabolism is used by the cell for synthesis of cell material and maintenance purposes. In other words, a certain fraction of the Gibbs free energy change has to be dissipated to allow the system to run. In a near-equilibrium system, a linear relationship between the rate of a process and its driving force is postulated (Rutgers et al., 1989). If the metabolism of $H$. foetida is viewed as a near-equilibrium system, the correlation found between growth kinetics and energetics can be understood on the basis of phenomenological non-equilibrium thermodynamics.

The influence of sulphide on growth under substrate limitation was investigated in continuous culture experiments. A dilution rate of $0.03 \mathrm{~h}^{-1}$ was chosen for all experiments. This value is low compared with the maximum growth rates in the presence of sulphide, and the system is, therefore, most likely energy-limited. It is also low enough to allow a steady-state growth in the 
absence of sulphide. It is, however, high enough to allow a valid comparison of growth parameters obtained in continuous culture with batch culture experiments in the absence of sulphide, as growth yield values can be influenced by the growth rate (Tempest \& Neijssel, 1984).

Growth yield values in the absence of sulphide in continuous culture were similar to those obtained in regulated batch cultures, and the stoichiometry of the metabolism suggests that the methyl groups were quantitatively metabolized through the acetyl-CoA pathway. However, in contrast to the results in regulated batch culture, sulphide addition did not significantly decrease growth yields in continuous culture. Together with the measured stoichiometry of the metabolism, this suggests that, under substrate limitation, a considerable fraction of the methyl groups is metabolized via the acetyl-CoA pathway with concomitant energy conservation, even though sulphide is present in the medium. It is unlikely that chemostat experiments selected for mutants well adapted to substrate-limited conditions, since batch culture experiments in the presence of sulphide performed with the same culture after continuous operation of the system yielded results similar to those obtained before continuous operation. A better explanation seems to be that the organism is able to modulate the flow of methyl groups into the two branches of methyl group metabolism depending on the environmental conditions. Whereas in a situation of excess substrate the methyl groups are quantitatively transferred to sulphide resulting in a high $-\Delta G_{\mathrm{D}}^{0 \prime}$ and a high specific growth rate but a low growth yield, a considerable fraction is channelled into the acetyl-CoA pathway under energy limitation. This results in a smaller $-\Delta G_{\mathrm{D}}^{\prime \prime}$, and a specific growth rate that is only slightly lower than in the absence of sulphide. Consequently, the specific substrate consumption rate shows only a minor increase due to sulphide addition.

The two branches of methyl group metabolism in $H$. foetida might be compared with the divided electron flow through the two NADH dehydrogenases (NDH-1 and NDH-2) in the respiratory chain of Escherichia coli. Here, electron flow to ubiquinone via $\mathrm{NDH}-2$, in contrast to NDH-1, is not coupled to proton translocation over the cytoplasmic membrane (Matsushita et al., 1987). Neijssel \& Teixeira de Mattos (1994) postulated that, under conditions of energy limitation, the maximal amount of protons should be translocated by predominant use of $\mathrm{NDH}-1$. Upon a change to conditions of excess substrate, however, NDH-2 would provide $E$. coli with a mechanism to decrease the energetic efficiency, thereby increasing the catabolic flux. This should result in an increased growth rate, the probably decisive factor under these conditions. However, to our knowledge, the electron flux through the two dehydrogenases under different environmental conditions has not been determined. Analogous to the considerations for the respiratory chain of E. coli, $H$. foetida conserves a greater part of the Gibbs free energy of the methyl group via the acetyl-CoA pathway, thereby minimizing the Gibbs free energy dissipated under conditions of substrate limitation. This could be a successful strategy to survive 'periods of famine' which are very common in nature. Under the rarer conditions of high substrate availability, $H$. foetida may transfer the methyl groups quantitatively to sulphide, thus lowering the growth yield and increasing Gibbs free energy dissipation of the overall metabolism and resulting in a marked increase in growth rate.

\section{ACKNOWLEDGEMENTS}

We sincerely thank Ralf Conrad for many valuable discussions and for critically reading the manuscript, and the reviewers for helpful comments on the manuscript. Markus Drescher's skilful construction and modification of various electronic devices used in this study is gratefully acknowledged. This investigation was supported by the Max-Planck-Gesellschaft, Germany.

\section{REFERENCES}

Bache, R. \& Pfennig, N. (1981). Selective isolation of Acetobacterium woodii on methoxylated aromatic acids and determination of growth yields. Arch Microbiol 130, 250-261.

Bak, F, Finster, K. \& Rothfuss, F. (1992). Formation of dimethylsulfide and methanethiol from methoxylated aromatic compounds and inorganic sulfide by newly isolated anaerobic bacteria. Arch Microbiol 157, 529-534.

Becher, B., Müller, V. \& Gottschalk, G. (1992). $N^{5}$-methyltetrahydromethanopterin: coenzyme $M$ methyltransferase of Methanosarcina strain Gö1 is a $\mathrm{Na}^{+}$translocating membrane protein. J Bacteriol 174, 7656-7660.

Brune, A. \& Schink, B. (1992). Phloroglucinol pathway in the strictly anaerobic Pelobacter acidigallici: fermentation of trihydroxybenzenes to acetate via triacetic acid. Arch Microbiol 157, $417-424$.

Cline, J. D. (1969). Spectrometric determination of hydrogen sulfide in natural waters. Limnol Oceanogr 14, 454-458.

Cypionka, H. (1986). Sulfide-controlled continuous culture of sulfate-reducing bacteria. J Microbiol Methods 5, 1-9.

van Dam, K., Mulder, M. M., Teixeira de Mattos, M. J. \& Westerhoff, H. V. (1988). A thermodynamic view of bacterial growth. In Physiological Models in Microbiology, vol. I, pp. 25-48. Edited by M. J. Bazin \& J. I. Prosser. Boca Raton, FL: CRC Press.

Drotar, A., Fall, L. R., Travernier, J. E. \& Fall, R. (1987). Enzymatic methylation of sulfide, selenite, and organic thiols by Tetrabymena thermophila. Appl Environ Microbiol 53, 2111-2118.

Finster, K., Tanimoto, Y. \& Bak, F. (1992). Fermentation of methanethiol and dimethylsulfide by a newly isolated methanogenic bacterium. Arch Microbiol 157, 425-430.

Frazer, A. C. (1994). O-Demethylation and other transformations of aromatic compounds by acetogenic bacteria. In Acetogenesis, pp. 445-483. Edited by H. L. Drake. New York, London: Chapman \& Hall.

Heijnen, J. J. (1994). Thermodynamics of microbial growth and its implications for process design. Tibtech 12, 483-492.

Heijnen, J. J. \& van Dijken, J. P. (1991). In a search of a thermodynamic description of biomass yields for the chemotrophic growth of microorganisms. Biotechnol Bioeng 39, 833-858. 
Kreft, J.-U. \& Schink, B. (1993). Demethylation and degradation of phenylmethylethers by the sulfide-methylating homoacetogenic bacterium strain TMBS 4. Arch Microbiol 159, 308-315.

Kreft, J.-U. \& Schink, B. (1994). O-Demethylation by the homoacetogenic anaerobe Holophaga foetida studied by a new photometric methylation assay using electrochemically produced cob(I)alamin. Eur J Biochem 226, 945-951.

Kreikenbohm, R. (1984). Untersuchungen von definierten Mischkulturen anaerober Bakterien in kontinuierlicher Kultur. PhD Thesis, University of Konstanz, Germany.

Krumböck, M. \& Conrad, R. (1991). Metabolism of positionlabelled glucose in anoxic methanogenic paddy soil and lake sediment. FEMS Microbiol Ecol 85, 247-256.

Liesack, W., Bak, F., Kreft, J.-U. \& Stackebrandt, E. (1994). Holophaga foetida gen. nov., sp. nov., a new, homoacetogenic bacterium degrading methoxylated aromatic compounds. Arch Microbiol 162, 85-90.

Matsushita, K., Ohnishi, T. \& Kaback, H. R. (1987). NADHubiquinone oxidoreductases of the Escherichia coli aerobic respiratory chain. Biochemistry 26, 7732-7737.

Mavrovouniotis, M. L. (1991). Estimation of standard Gibbs energy changes of biotransformations. $J$ Biol Chem 266, $14440-14445$

Neijssel, O. M. \& Teixeira de Mattos, M. J. (1994). The energetics of bacterial growth : a reassessment. Mol Microbiol 13, 179-182.

Pirt, S. J. (1965). The maintenance energy of bacteria in growing cultures. Proc R Soc Lond Ser B 163, 224-231.

Rutgers, M., Balk, P. A. \& van Dam, K. (1989). Effect of concentration of substrates and products on the growth of Klebsiella pneumoniae in chemostat cultures. Biochim Biophys Acta 977, 142-149.
Schink, B. \& Pfennig, N. (1982). Fermentation of trihydroxybenzenes by Pelobacter acidigallici gen. nov. sp. nov., a new strictly anaerobic, non-sporeforming bacterium. Arch Microbiol 133, 195-201.

Stucki, J. W. (1980). The optimal efficiency and the economic degrees of coupling of oxidative phosphorylation. Eur J Biochem 109, 269-283.

Teixeira de Mattos, M. J. \& Tempest, D. W. (1983). Metabolic and energetic aspects of the growth of Klebsiella aerogenes NCTC 418 on glucose in anaerobic chemostat culture. Arch Microbiol 134, $80-85$.

Tempest, D. W. \& Neijssel, O. M. (1984). The status of $Y_{A T P}$ and maintenance energy as biologically interpretable phenomena. Annu Rev Microbiol 38, 459-486.

Thauer, R. K., Jungermann, K. \& Decker, K. (1977). Energy conservation in chemotrophic anaerobic bacteria. Bacteriol Rev 41, 100-180.

Tschech, A. \& Pfennig, N. (1984). Growth yield increase linked to caffeate reduction in Acetobacterium woodii. Arch Microbiol 137, 163-167.

de Vries, W., Kapteijn, W. M. C., van der Beek, E. G. \& Stouthamer, A. H. (1970). Molar growth yields and fermentation balances of Lactobacillus casei L3 in batch cultures and in continuous cultures. J Gen Microbiol 63, 333-345.

Westerhoff, H. V. \& van Dam, K. (1987). Thermodynamics and Control of Biological Free-energy Transduction. Amsterdam: Elsevier.

Received 24 July 1996; revised 27 November 1996; accepted 3 December 1996. 